\title{
Bovine Serum Albumin and Fibrinogen Adsorption at the 316L Stainless Steel/Aqueous
}

\section{Interface}

Mary H. Wood, ${ }^{1 *}$ Charanee Galabada Payagalage, ${ }^{1}$ Thomas Geue ${ }^{2}$

${ }^{1}$ Department of Chemistry, University of Cambridge, Lensfield Road, Cambridge, CB2 1EW,

UK

${ }^{2}$ Laboratory of Neutron Scattering and Imaging, Paul Scherrer Institute, 5232 Villigen PSI, Switzerland

*maryhwood@gmail.com (+44)1214 142966

This document is the accepted manuscript version of the following article: Wood, M. H., Payagalage, C. G., \& Geue, T. (2018). Bovine serum albumin and fibrinogen adsorption at the $316 \mathrm{~L}$ stainless stee1/aqueous interface. Journal of Physica1 Chemistry B, 122(19), 5057-5065. https://doi.org/10.1021/acs.jpcb.8b01347 
ABSTRACT: The binding of bovine serum albumin (BSA) to a $316 \mathrm{~L}$ stainless steel surface from a buffer solution has been characterized using neutron reflectometry (NR) and quartz crystal microbalance (QCM) measurements; coverage at all concentrations up to a nearphysiological concentration was found to be relatively low (<20\%); the protein followed a two-step isotherm adsorption model type and the overall thickness at the higher concentrations (around $80 \AA$ ) suggested possible multi-layering and/or protein unfolding. As it has been postulated that BSA may inhibit the further adsorption of another blood plasma protein-fibrinogen - the effects of pre-adsorbing BSA on fibrinogen adsorption were examined, first by prior physisorption of BSA to the stainless steel surface and second by pre-treating the stainless steel with a layer of SDS to render it more hydrophobic. While the pre-adsorption of BSA to an untreated stainless steel surface did slightly decrease the amount of fibrinogen adsorbed initially, it had no inhibiting effect if a solution containing solely fibrinogen subsequently flowed through. In contrast, the SDS-treated surface yielded both an increased BSA adsorption and consistently decreased fibrinogen adsorption. 


\section{INTRODUCTION}

Understanding the interaction of potential biomaterials with physiological proteins is extremely important when considering their biocompatibility. One of the most persisting concerns when introducing a new material into the body is the possible onset of thrombosis, whereby uncontrollable clotting at the surface results in large emboli that may break away and block blood veins with potentially fatal consequences. The formation of blood clots via coagulation is an extremely complex process and is considered to follow two possible pathways - the extrinsic pathway, which is initiated when trigger chemicals are released from damaged blood vessel walls, and the intrinsic pathway, which is initiated by platelet damage or by contact of the blood with a foreign body such as a biomaterial. Traditionally, it has been thought that the extrinsic pathway plays no role in coagulation resulting from biomaterial introduction, although recent studies have shed some doubt on this view ${ }^{1}$. The exact mechanisms of biomaterial-induced coagulation are not yet completely understood and appear to rely on a great number of factors. However, it is well-established that any surface will be coated in a layer of proteins within seconds of encountering the blood plasma, and that this adsorbed protein layer will be critical in determining ensuing success or rejection of the biomaterial ${ }^{2}$.

As fibrinogen plays a direct role in the coagulation process, it is unsurprising that its interaction with biomaterials has been of particular interest, and indeed platelet adhesion to the biomaterial has been found to be dependent both on amount on fibrinogen adsorbed and, crucially, on its adsorbed state conformation, which is dependent on the surface characteristics of the biomaterial ${ }^{1,3-5}$. Whilst fibrinogen is not the most abundant protein in the blood plasma, it is often the most extensively adsorbed across a range of surface types 
(although the Vroman effect may subsequently diminish its surface presence) ${ }^{6-8}$; hence, a surface that may be considered 'invisible' to the fibrinogen protein has long been sought. One commonly-used strategy is to pre-treat the biomaterial surface with serum albumin. This is the most abundant blood plasma protein and is not involved in the coagulation process, its function being rather the transport of fatty acids and other species ${ }^{9}$. Preadsorption of albumin is thought to prevent subsequent adsorption of coagulation proteins such as fibrinogen and hence to render the surface more biocompatible ${ }^{10-12}$. As albumin only binds weakly to most surfaces, particularly when in competition with other plasma proteins, it is necessary to pre-bind the albumin before exposure to physiological fluids ${ }^{11,13}$. Indeed, some studies have found that it is only significantly effective if the physisorption is enhanced by making the surface more hydrophobic, or if the albumin is cross-linked itself when adsorbed ${ }^{14,15}$. One disadvantage of the albumin pre-treatment is that adsorbed proteins tend to denature over time or to desorb; as many implants need to remain in the body safely for a long duration, a surface treatment that will endure over a long timescale is crucial $^{10}$. Increasing the hydrophobicity of the surface has been found to improve the longevity of albumin-coated devices; this effect is presumed to arise both from an increase in the amount adsorbed, and also from the increased unfolding and relaxation of the albumin protein molecules, such that their hydrophobic domains are able to interact with the surface, leading to an overall increased surface footprint ${ }^{16-19}$. Uyen et al. observed that islands of albumin that adsorb initially onto hydrophobic surfaces grow more over time than on hydrophilic surfaces; they related this to increased lateral interactions between the adsorbed albumin molecules ${ }^{20}$. In addition, it has been shown that BSA adsorption to various grades of stainless steel, including the $316 \mathrm{~L}$ surgical grade used in this study, is liable to induce significant release of metal ions into solution ${ }^{21}$. As the corrosion of biomedical 
implants is extremely detrimental to their usability, surface modification to protect the stainless steel over a long period of implantation is clearly advisable.

In this work, the adsorption of bovine serum albumin (BSA) on a 316L stainless steel surface was measured and its effect on further adsorption of fibrinogen monitored using both neutron reflectometry (NR) and quartz crystal microbalance (QCM). NR was also used to assess the efficacy of pre-coating the stainless steel surface with sodium dodecyl sulfate (SDS) - in an attempt to render it more hydrophobic-on both improving BSA adsorption and hindering fibrinogen adsorption. NR is a powerful technique for monitoring the structure of buried interfacial layers, particularly for organic species that are harder to detect using $\mathrm{X}$-rays ${ }^{22}$. Full details of the underlying theory for the technique may be found elsewhere ${ }^{23,24}$. The NR data are modelled to give a profile of the scattering length density (SLD) as a function of the distance along the surface normal, z. SLD values of the materials of interest in this work are given in Table 1 . We have previously demonstrated that by using an electron-beam deposition technique, a film of stainless steel can be successfully deposited onto a silicon substrate to provide a model stainless steel surface that is suitable for NR experiments ${ }^{25}$. Here, we extend the technique to a more complex protein system. In addition, QCM allows measurement of protein adsorption at the solid/liquid interface in real time via changes in the frequencies of a resonant crystal beneath the stainless steel layer ${ }^{26}$.

Table 1. SLD Values. 'BSA' = bovine serum albumin. The SLD of the protein is dependent on the solvent contrast, due to its large number of exchangeable protons.

\begin{tabular}{cccc}
\hline & $\mathrm{SLD} / \times 10^{-6} \AA^{-2}$ & $\mathrm{SLD} / \times 10^{-6} \AA^{-2}$ \\
\hline $\mathrm{Si}$ & 2.072 & $\mathrm{Fe}_{2} \mathrm{O}_{3}$ & 7.176 \\
$\mathrm{SiO}_{2}$ & 3.484 & $\mathrm{Cr}_{2} \mathrm{O}_{3}$ & 5.106 \\
$\mathrm{Fe}$ & 8.020 & $\mathrm{H}_{2} \mathrm{O}$ & -0.561 \\
$\mathrm{Cr}$ & 3.027 & $\mathrm{D}_{2} \mathrm{O}$ & 6.335 \\
$\mathrm{Ni}$ & 9.406 & $\mathrm{BSA} / 100 \% \mathrm{H}_{2} \mathrm{O}$ & 1.853 \\
$\mathrm{Mn}$ & -3.054 & $\mathrm{BSA} / 100 \%$ & 3.056 \\
& \multicolumn{4}{c}{} \\
\hline
\end{tabular}




\section{EXPERIMENTAL}

Materials. For the NR experiments, $316 \mathrm{~L}$ stainless steel films (10-20 nm thickness) were deposited onto polished silicon substrates, (111)-orientation, (n)-type, using electronbeam deposition in vacuo at the Nanoscience Centre at the University of Cambridge. 316 stainless steel QCM sensors were purchased from Q-sense. Fibrinogen and Bovine serum albumin were purchased from MP Biomedicals and all other chemicals were acquired from Sigma-Aldrich (all with purities $>99 \%$ ). All experiments were conducted in phosphate buffered saline ('PBS', $0.01 \mathrm{M}, \mathrm{pH} 7.4$ ).

XRR. XRR profiles were collected at the Cavendish Laboratory, Cambridge using a Bruker D8 X-ray diffractometer with copper target and a Goebel mirror. An accelerating voltage of $50 \mathrm{kV}$ was used and a primary beam size of $0.1 \mathrm{~mm} .0 .35 \mathrm{~mm}$ Soller slits were inserted before the detector, which was operated in 1D mode. XRR data were fitted using GenX 2.0.0 software ${ }^{27}$.

NR. Neutron reflectometry data were collected using the AMOR instrument at the Paul Scherrer Institute in Switzerland. Full details of the instrument may be found elsewhere ${ }^{28}$. The instrument was used in non-polarised mode; stainless steel-coated silicon blocks ( $55 \mathrm{~mm}$ diameter, $5 \mathrm{~mm}$ thick) were mounted in a custom-made aluminium cell with a PTFE trough. The bare substrates were characterised in $\mathrm{D}_{2} \mathrm{O}$ and $\mathrm{H}_{2} \mathrm{O}$ prior to introduction of the protein solutions. Sample 2 was pre-coated with an SDS layer by soaking in an SDS/ethanol solution (7 mM) for 24 hours before rinsing and drying under $\mathrm{N}_{2}$ prior to mounting in the sample cell. NR data were fitted using the RasCAL software ${ }^{29}$. 
QCM. The 316 stainless steel sensors were cleaned prior to use in $1 \%$ Hellmanex II (30 min), rinsed with ultrapure water $(18 \mathrm{M} \Omega \mathrm{cm})$ and dried with $\mathrm{N}_{2}$; they were then sonicated in $99 \%$ ethanol (10 $\mathrm{min})$, and rinsed and dried again before cleaning by UV/ozone (10 min). Experiments were conducted on a Q-sense E4 QCM at the Nanoscience Centre, University of Cambridge. The QCM instrument was cleaned with 2\% Hellmanex and rinsed thoroughly with ultrapure water before loading of the sensors. Background solution (PBS) was flowed through and the sensors left for $24 \mathrm{~h}$ to equilibrate. Fresh electrolyte was then flowed through until the frequencies had stabilized. The sample solutions were then introduced to each sensor (flow rate $0.15 \mathrm{~mL} \mathrm{~min}^{-1}$ ) until the frequencies were stable. After the systems had reached equilibrium, background electrolyte was reintroduced to each one until no further changes were observed. Data were analyzed using the QSense Dfind 1.0 software. For Sauerbrey model fits, the $\mathrm{f} 3$ overtone was used; for viscoelastic model fits, a Voigt model was used.

\section{RESULTS AND DISCUSSION}

NR.

Sample 1. 

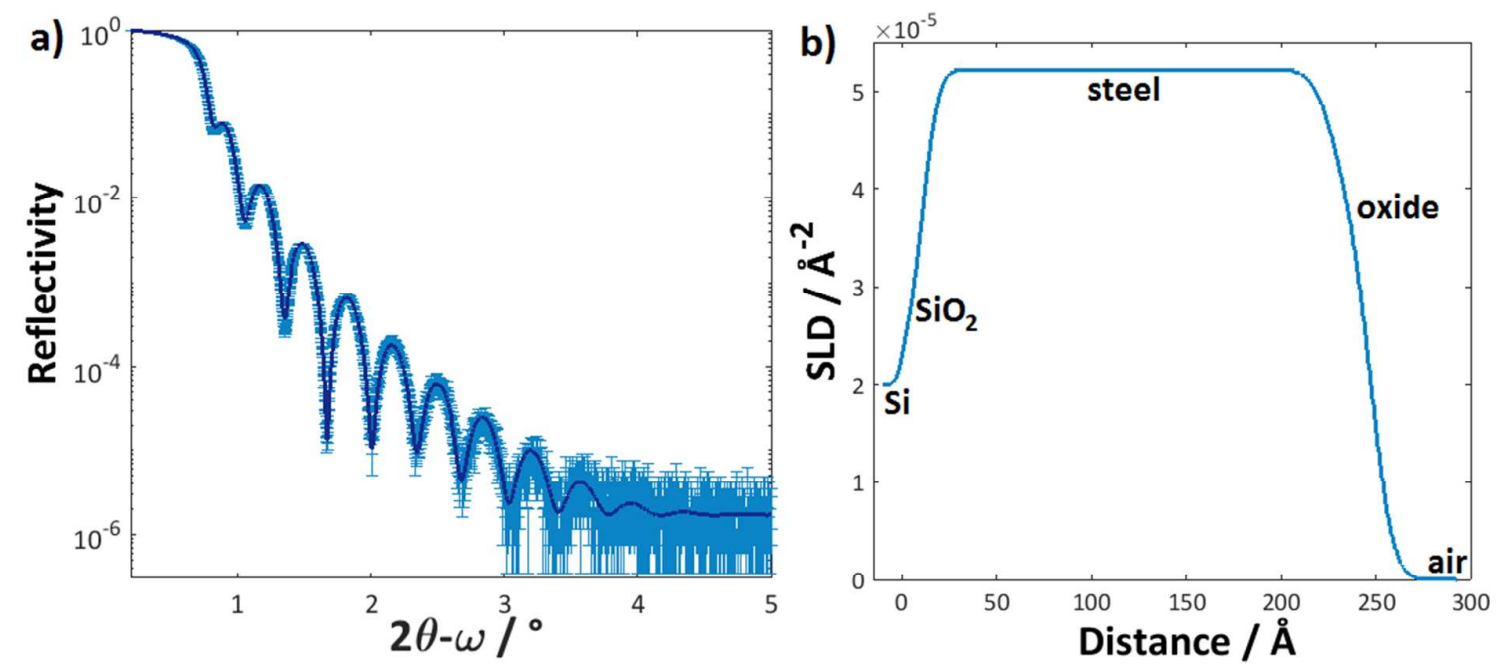

Figure 1a) XRR data (points) and model fits (solid lines) for a stainless steel film on a silicon substrate (Sample 1). b) Corresponding SLD profiles for the model fit.

Sample 1 was characterized using XRR prior to analysis by NR; the data and model fits are shown in Figure 1. The data were fitted with just one metal layer for the stainless steel of thickness $217 \AA( \pm 2 \AA)$ and an oxide layer of thickness $21 \AA( \pm 2 \AA)$, in good agreement with the estimated deposition thickness values. (Error margins taken from limits of reasonable fit.) 

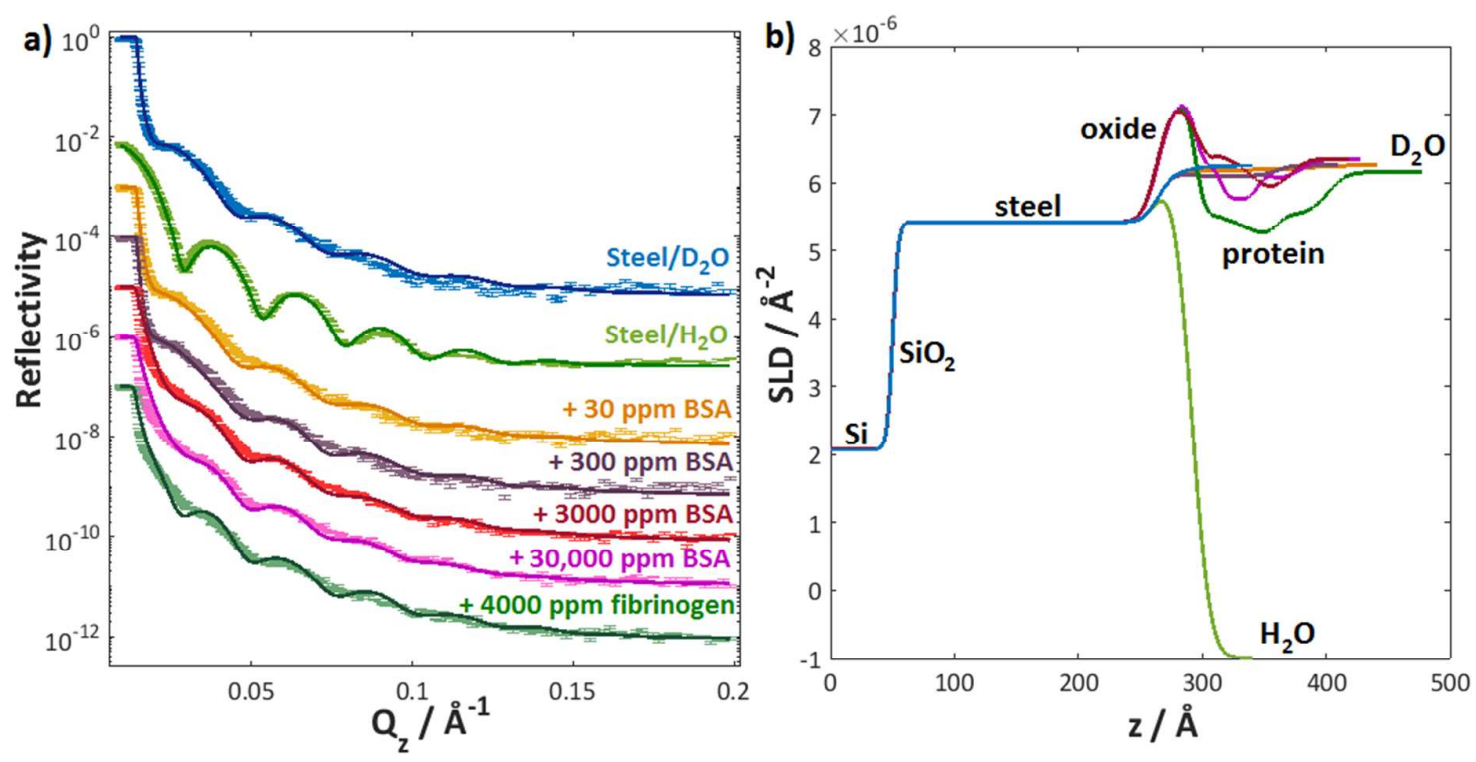

Figure 2a) Neutron reflectometry data (points) and model fits (solid lines) for sample 1, showing the untreated stainless steel surface under $\mathrm{D}_{2} \mathrm{O}$ and $\mathrm{H}_{2} \mathrm{O}$ and with increasing concentrations of protein added, as labelled. $\left(D_{2} \mathrm{O}\right.$ and $\mathrm{H}_{2} \mathrm{O}$ are given as shorthand for $\mathrm{PBS} / \mathrm{D}_{2} \mathrm{O}$ and $\mathrm{PBS} / \mathrm{H}_{2} \mathrm{O}$ ). Data offset vertically for clarity. Only $\mathrm{D}_{2} \mathrm{O}$ data are shown for the protein solutions as these show the greatest contrast. b) Corresponding SLD profiles for the model fits.

The NR data and model fits for the untreated stainless steel sample are shown in Figure 2; the stainless steel film under PBS/water could be modelled with a metal layer of thickness $214 \AA( \pm 2 \AA)$, roughness $15 \AA$ and SLD $5.42 \times 10^{-6} \AA^{-2}$, and an oxide layer of thickness $30 \AA$ ( $\pm 3 \AA$ ) and SLD $6.15 \times 10^{-6} \AA^{-2}$. The thicknesses are in good agreement with the XRR data (the increase in oxide thickness is attributed to the UV/ozone cleaning prior to NR measurements) and the SLD values are similar to those seen previously ${ }^{25}$, with the oxide SLD consistent with that expected for a mixture of iron and chromium oxides (values for pure $\mathrm{Cr}_{2} \mathrm{O}_{3}$ and $\mathrm{Fe}_{2} \mathrm{O}_{3}$ would be 5.11 and $7.17 \times 10^{-6} \AA^{-2,}$ respectively). While the SLD of $316 \mathrm{~L}$ stainless steel should theoretically be closer to $7 \times 10^{-6} \AA^{-2}$, an increased amount of $\mathrm{Mn}$ 
(which has a very low SLD) was previously observed in the deposited films, hence decreasing the overall SLD ${ }^{25}$.

Table 2. Adsorbed Amounts Calculated from Neutron Fits for Both Samples Error margins are calculated from the maximum and minimum acceptable fits to the NR data. *Surfaces finally washed with PBS (no protein) following adsorption.

\begin{tabular}{cccc}
\hline & $\begin{array}{c}\text { [BSA] } \\
\text { / ppm }\end{array}$ & $\begin{array}{c}\text { [fibrinogen] } \\
/ \text { ppm }\end{array}$ & $\begin{array}{c}\text { Amount adsorbed } \\
/ \mathrm{mg} \mathrm{m}^{-2}\end{array}$ \\
\hline & 30 & 0 & $0.25( \pm 0.2)$ \\
Sample 1 & 300 & 0 & $0.58( \pm 0.4)$ \\
& 3000 & 0 & $0.74( \pm 0.4)$ \\
& 30000 & 0 & $1.22( \pm 0.4)$ \\
& 30000 & 4000 & $3.35( \pm 0.4)$ \\
& 0 & 4000 & $4.75( \pm 0.4)$ \\
Sample 2 & $0^{*}$ & $0^{*}$ & $3.53( \pm 0.4)$ \\
(pre-treated with & 30000 & 0 & $1.77( \pm 0.4)$ \\
SDS) & 30000 & 4000 & $2.29( \pm 0.4)$ \\
& 0 & 4000 & $3.64( \pm 0.4)$ \\
& $0^{*}$ & $0^{*}$ & $2.90( \pm 0.4)$ \\
\hline
\end{tabular}

Only very slight changes in the measured data were seen after the addition of 30 ppm and 300 ppm BSA; these could be simply modelled by inclusion of a Gaussian protein layer. For the 3000 and 30,000 ppm BSA, a Gaussian layer was unable to satisfactorily fit the data; instead, a block model with several layers was used, where the \% of hydration (i.e. extent of solvent inclusion in the protein layer) was allowed to vary for each layer. The overall protein thicknesses for 3000 and 30,000 ppm were $75 \AA$ and $83 \AA$ respectively. Using either the area under the Gaussian curve or the area encapsulated by the block models as appropriate, the amount of protein adsorbed per square metre of the substrate was calculated and used to infer an adsorption isotherm for BSA on a 316L stainless steel surface, shown in Figure 3 ( $x$-axis plotted as a log scale for clarity). The fitted amounts adsorbed are given in Table 2 . 


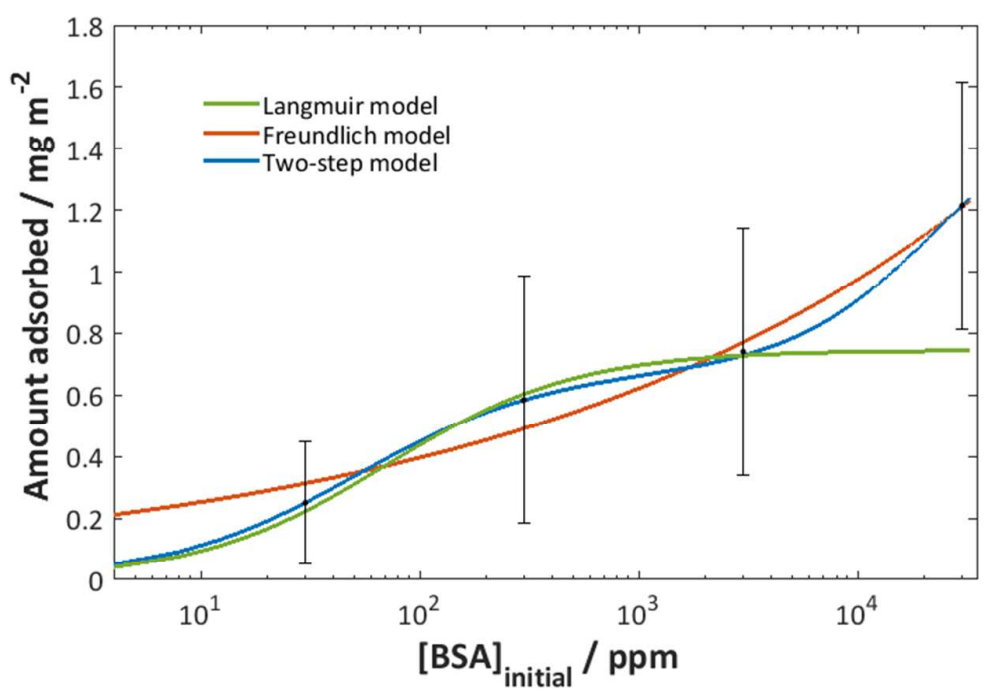

Figure 3. Isotherm calculated from the fitted neutron reflectometry data for increasing concentrations of BSA on the untreated stainless steel surface. Data are plotted as points with model fits as solid lines, as labelled. Error margins are calculated from the limits of a reasonable fit to the neutron data.

Up to and including 3000 ppm BSA, a simple Langmuir model was sufficient to fit the data; however, the data point at 30,000 ppm did not fit this trend. Two alternative isotherm models were fitted to the data, as shown in Figure 3. The Freundlich isotherm model gave an more acceptable fit to the data; this model assumes ongoing interaction between the adsorbed protein and incoming protein molecules, given by the equation:

$$
v=v_{\max } K_{F} c^{\frac{1}{n}}
$$

where $v$ is the fraction adsorbed, $v_{\max }$ is the final plateau of adsorption, $K_{F}$ is the affinity parameter, $c$ is the adsorbate concentration and $n$ is a dimensionless constant $>1$ that relates to the strength of adsorbate interactions with either the substrate or other adsorbate molecules ${ }^{30,31}$. This would not be an unreasonable assumption for protein adsorption, since proteins are known to change in structure upon adsorption to solid 
surfaces, and the large number of functional groups on any protein makes interaction with other adsorbing proteins feasible.

The two-step isotherm model was proposed by Zhu et al. and assumes Langmuir-like behavior at low concentrations, but with a second plateau at higher concentrations; they found this model described systems in which monomer adsorption at low concentrations was followed by adsorption of aggregated molecules at higher concentrations ${ }^{32}$. It has also been used previously to describe protein adsorption due to the potential protein-protein interactions in the adsorbed layers ${ }^{25}$. The isotherm model is given by:

$$
v=\frac{v_{\max } K_{1} c\left(\frac{1}{n}+K_{2} c^{n-1}\right)}{1+K_{1} c\left(1+K_{2} c^{n-1}\right)}
$$

where $K_{1}$ and $K_{2}$ are affinity parameters for the first and second steps respectively. As shown in Figure 3, the two-step model gives the best fit to the isotherm data; however, this might be expected for the greater number of parameters that can be fitted, particularly given the relatively large error bars associated with these measurements.

Both the Freundlich and two-step isotherm models give acceptable fits to the experimental data, indicating that at higher protein concentrations some kind of interaction with the protein already adsorbed may lead to increased adsorption, possibly in the form of a bilayer; this would agree well with the increased layer thicknesses seen at higher concentrations (as shown in the SLD profile in Figure 2b) of 75 and $83 \AA$ at 3,000 and 30,000 ppm. The dimensions of a hydrated bovine serum albumin molecule have been estimated ${ }^{33}$ as $140 \AA$ × $40 \AA$ x $40 \AA$; a bilayer in which the larger dimension is lying parallel to the surface would, therefore, be consistent with the fitted data for the higher protein concentrations, and accord well with the fitted isotherm models. Similar multi-step behavior has been previously observed for albumin at the stainless steel/buffer solution interface and ascribed 
to various protein-protein interactions within the adsorbed film, although further more specific measurements would be necessary to confirm this ${ }^{34,35}$.

Assuming a volume per molecule of $\mathrm{BSA}^{36}$ of around $1.08 \times 10^{5} \AA^{2}$, and treating the protein molecules as spheres, each molecule might be expected to occupy approximately $5500 \AA^{2}$ on the stainless steel surface. The areas per molecule calculated from the values in Table 2 would yield an area per molecule of $15000 \AA^{2}$ at 3000 ppm and $9000 \AA^{2}$ at 30,000 ppm. This is in good agreement with the low surface coverage suggested by the model fit to the neutron data, where a large amount of water inclusion was necessary for each layer $(\geq$ $80 \%)$.

The adsorbed amount for the BSA/316L stainless steel system across this concentration range is significantly lower than that seen for an isotherm of fibrinogen previously derived from NR data ${ }^{25}$. (This trend is also observed when both isotherms are plotted in terms of molar concentration, as demonstrated in Figure S1.) The Langmuir plateau value seen here is very similar to that observed previously for BSA adsorbed onto $316 \mathrm{~L}$ stainless steel particles for comparable protein concentrations around $\mathrm{pH} 7^{37,38}$. A higher adsorption coverage was seen by Van Enckevort et al. for a 304 stainless steel slide measured using ${ }^{125}$ I-labelled albumin ${ }^{39}$; however, use of isotopic labelling is known to lead to overestimation of adsorption coverages, due to free ${ }^{125}$ I that remains associated with the surface $e^{34,40}$. The slight differences in grade of stainless steel may also account for differences in the protein surface coverage, particularly since stainless steel particles are known to be enriched in manganese, similarly to the deposited films used here ${ }^{41}$.

When a solution of 30,000 ppm BSA plus 4000 ppm fibrinogen was subsequently flushed through the cell, the overall amount of protein adsorbed increased to $3.0 \mathrm{mg} \mathrm{m}^{-2} \Psi^{ \pm}$ $0.4 \mathrm{mg} \mathrm{m}^{-2}$ ). This is less than the amount adsorbed calculated from NR data for fibrinogen 
on its own on a $316 \mathrm{~L}$ stainless steel surface observed previously $-4.7 \mathrm{mg} \mathrm{m}^{-2}$ at $4000 \mathrm{ppm}$ fibrinogen ${ }^{25}$. Even within the relatively large error bars of the experiment, this reflects a significant enough decrease to indicate that the adsorbed BSA does prevent fibrinogen adsorption to some extent. However, when the cell was flushed through with a solution of fibrinogen alone (4000 ppm), the adsorbed amount increased to $4.75 \mathrm{mg} \mathrm{m}^{-2}\left( \pm 0.4 \mathrm{mg} \mathrm{m}^{-2}\right)$. This almost exactly matches the value seen previously when fibrinogen was allowed to adsorb on stainless steel that had not previously encountered BSA. It would therefore seem that the BSA only has a preventative effect on the fibrinogen adsorption if it remains the dominant species in the bulk solution, and is presumably quickly displaced from the surface by the fibrinogen.

Wertz et al. put forward the interesting theory that the adsorption of albumin is greatly dependent on the rate at which it arrives at the surface-they posit that when the rate is slow, small amounts of albumin that arrive initially and adsorb in their molecular form will unfold and spread across the entire surface, preventing further adsorption, whereas rapidly-arriving albumin (i.e. from high initial bulk concentrations) will adsorb in larger amounts as there is no time for this unfolding ${ }^{42}$. However, the results shown here would seem to contradict this theory, at least to some extent, as the increasing concentrations of albumin were flowed over the same sample without rinsing in between, and as each measurement took in excess of 6 hours to complete, there should have been ample time for such unfolding-however, there does not seem to be any inhibition of further albumin adsorption at the higher concentrations, which suggests that the protein adsorbed at lower concentrations has not altered the surface significantly enough to prevent further adsorption. These contrasting observations to those of Wertz et al. may arise from the differences in surface type, as their measurements were conducted on a 
hydrophobic, C16 self-assembled monolayer surface, whereas the negatively-charged stainless steel sample used in this work may be less conducive to protein unfolding, given that the albumin molecules are also expected to be negatively-charged at this $\mathrm{pH}^{21}$. This further highlights the importance of surface preparation with respect to biomaterial design.

Sample 2. In an attempt to increase the BSA coverage, and therefore decrease fibrinogen adsorption, a second stainless steel sample was pre-treated with an SDS/ethanol solution in the hope of pre-adsorbing a layer of SDS and hence rendering the surface more hydrophobic. The data and model fits are shown in Figure 4; similarly to sample 1, a stainless steel layer $211 \AA$ thick $\left( \pm 3 \AA\right.$ ) with SLD $5.5 \times 10^{-6} \AA^{-2}$ and roughness $15 \AA$ and an oxide layer $25 \AA$ thick ( $\pm 3 \AA$ ) with SLD $7.1 \times 10^{-6} \AA^{-2}$ were found to give a good fit to the data. In addition, an SDS layer (SLD $\left.0.34 \times 10^{-6} \AA^{-2}\right) 30 \AA$ ( $\pm 3 \AA$ ) thick was found to have adsorbed with $46 \%$ coverage (as seen by the significant dip in the SLD profile adjacent to the stainless steel surface in Figure 4b). This demonstrated that the pre-soaking of the stainless steel substrate in the SDS/ethanol solution had been successful in yielding a surface layer about half of that expected for a fully well packed surfactant layer.
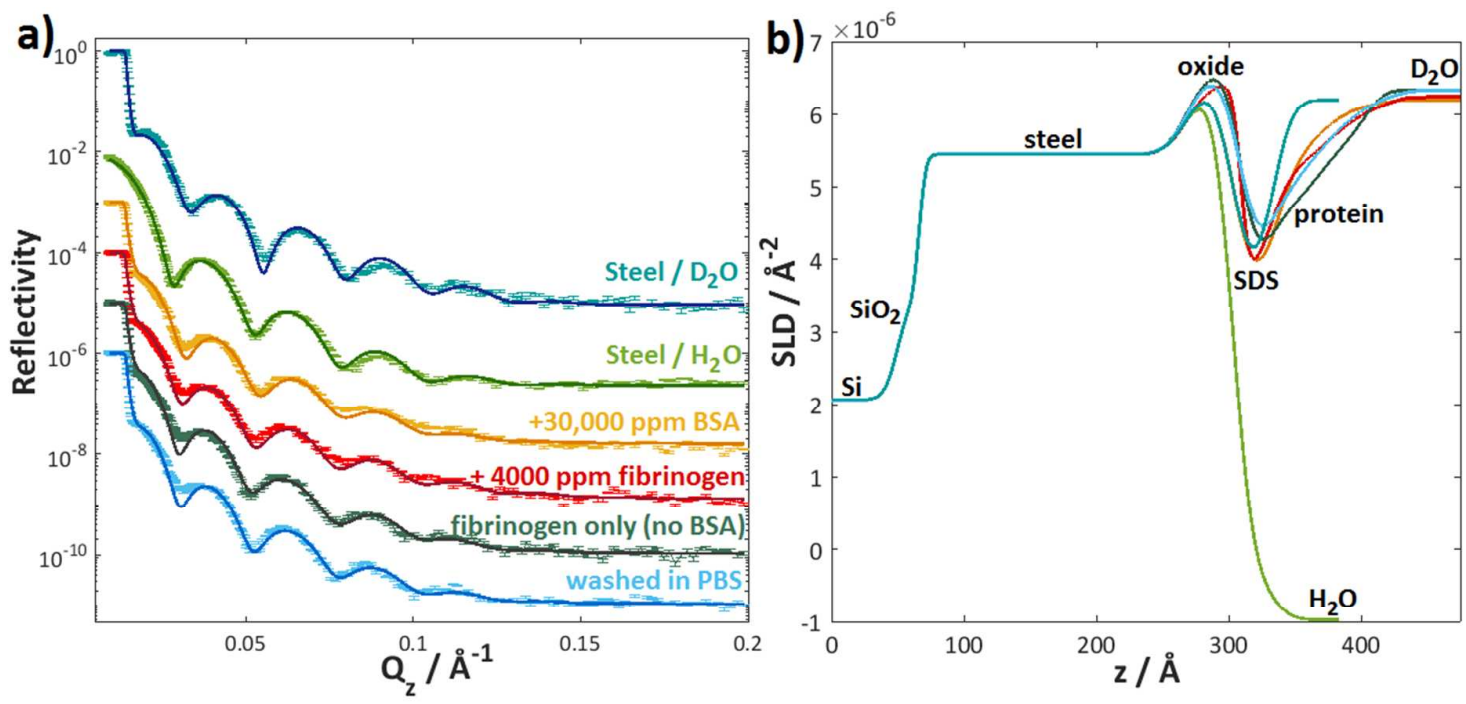


\section{Figure 4a) Neutron reflectometry data (points) and model fits (solid lines) for sample 2, showing the untreated stainless steel surface under $\mathrm{D}_{2} \mathrm{O}$ and $\mathrm{H}_{2} \mathrm{O}$ and with increasing concentrations of protein added, as labelled. Data offset vertically for clarity. Only $D_{2} O$ data are shown for the protein solutions as these show the greatest contrast. b) Corresponding SLD profiles for the model fits.}

Upon addition of BSA, a Gaussian model was again used to fit the adsorbed layer, with calculated amounts adsorbed summarized in Table 2. At a concentration of 30,000 ppm BSA, an increase in the amount of protein adsorbed in comparison to Sample 1 was observed, suggesting the SDS did indeed facilitate BSA adsorption. Significantly lower amounts of adsorbed protein in comparison to Sample 1 were seen upon subsequent addition of fibrinogen, both with BSA and by itself. When 4000 ppm fibrinogen was flushed through the cell, the amount adsorbed was $3.64 \mathrm{mg} \mathrm{m}^{-2}\left( \pm 0.4 \mathrm{mg} \mathrm{m}^{-2}\right)$, which is considerably less than that seen for fibrinogen on the bare stainless steel substrate ${ }^{25}$. While the different proteins cannot be distinguished from this data as both were in protonated form with similar SLD values $\left(3.06 \times 10^{-6} \AA^{-2}\right.$ for BSA and $3.26 \times 10^{-6} \AA^{-2}$ for fibrinogen in 100 $\% D_{2} O$ ), the natural conclusion is that the presence of the SDS is ensuring more BSA is retained on the surface with a subsequent inhibitory effect on fibrinogen adsorption. High concentrations of SDS have previously been thought to have a partially denaturing effect on albumin pre-adsorbed onto $316 \mathrm{~L}$ stainless steel surfaces ${ }^{43}$. It is possible that the strength of the SDS-albumin interaction may also be causing some unfolding in this case, which may contribute to the prevention of fibrinogen adsorption.

Given how low the overall protein coverage is in each case, it is interesting that the small amounts of BSA adsorbed make any difference at all, since the incoming fibrinogen 
might be expected to simply bind in the spaces left by the BSA. However, it is well known that in general, adsorption of surfactant molecules may occur preferentially at certain more 'active' metal surface sites ${ }^{44-46}$; as the NR technique yields structural information averaged in the z-direction, we cannot draw any conclusions concerning the specific locations of the SDS/BSA adsorption, but it may be that even a relatively low coverage is sufficient to block sites at which fibrinogen preferentially interacts with the stainless steel surface and hence inhibit its adsorption.

For both samples, a significant amount of protein was left on the surface after washing with PBS solutions containing no dissolved protein (Table 2, represented by protein concentrations of ' $0 \mathrm{ppm}$ '), confirming that the adsorption is at least partially irreversible, as has been observed for both fibrinogen and BSA on various surfaces previously ${ }^{38,47}$, and in good agreement with the QCM results to be presented below.

\section{QCM.}

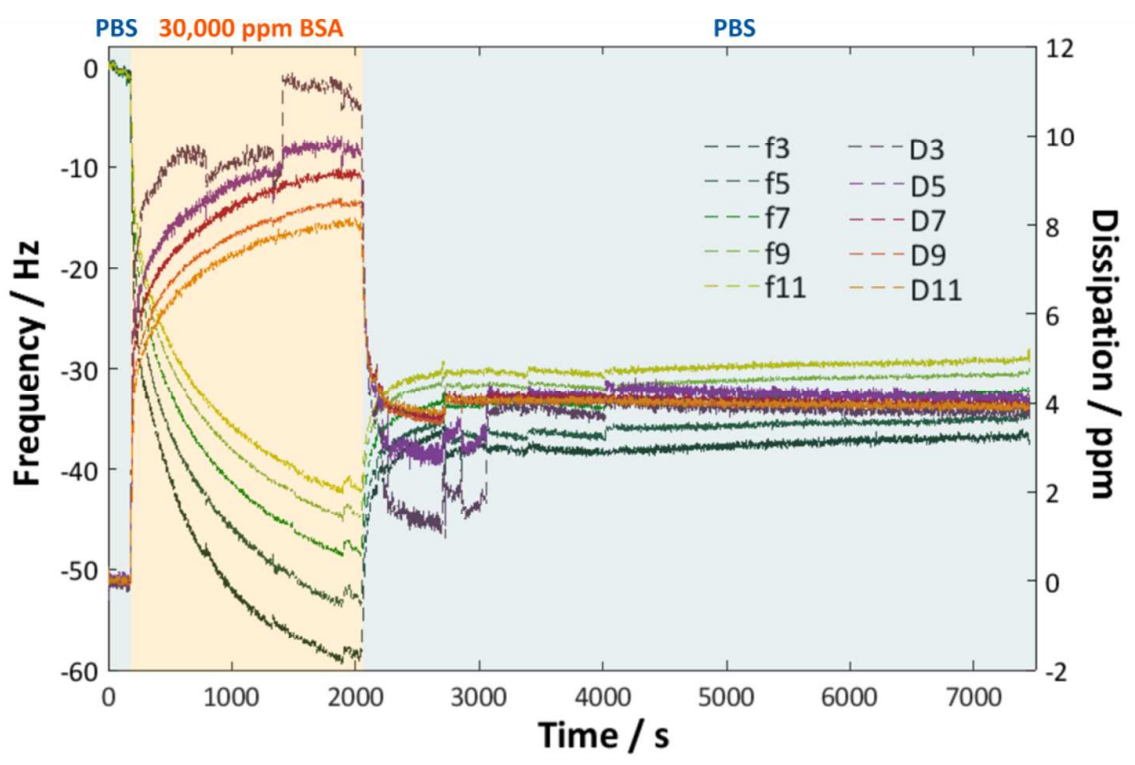

Figure 5. QCM frequency and dissipation data for 316 stainless steel sensor under PBS

(blue), with 30,000 ppm BSA added (orange) and flushed again with PBS (blue). 
Figure 5 shows the variation in frequency and dissipation for a 316 stainless steel sample after exposure to $30,000 \mathrm{ppm}$ BSA/PBS, and then following rinsing in PBS, as marked on the plot. It is clear that a significant amount of protein was adsorbed, and that although the PBS rinse removed roughly a third of this adsorbed protein essentially immediately, the rest was stable over a prolonged period of measurement.

The separation of the frequency measurements for the different fundamentals and the variable dissipation suggest the adsorbed protein cannot be treated as a simple, rigid layer as assumed by the Sauerbrey model, although the layer appears to become more Sauerbrey-like after the rinse, as evidenced by the frequency lines moving closer together. A viscoelastic model gave a reasonable fit to the data (Figure S2) and the adsorbed layer thicknesses and masses predicted by the different models are compared in Figure 6.
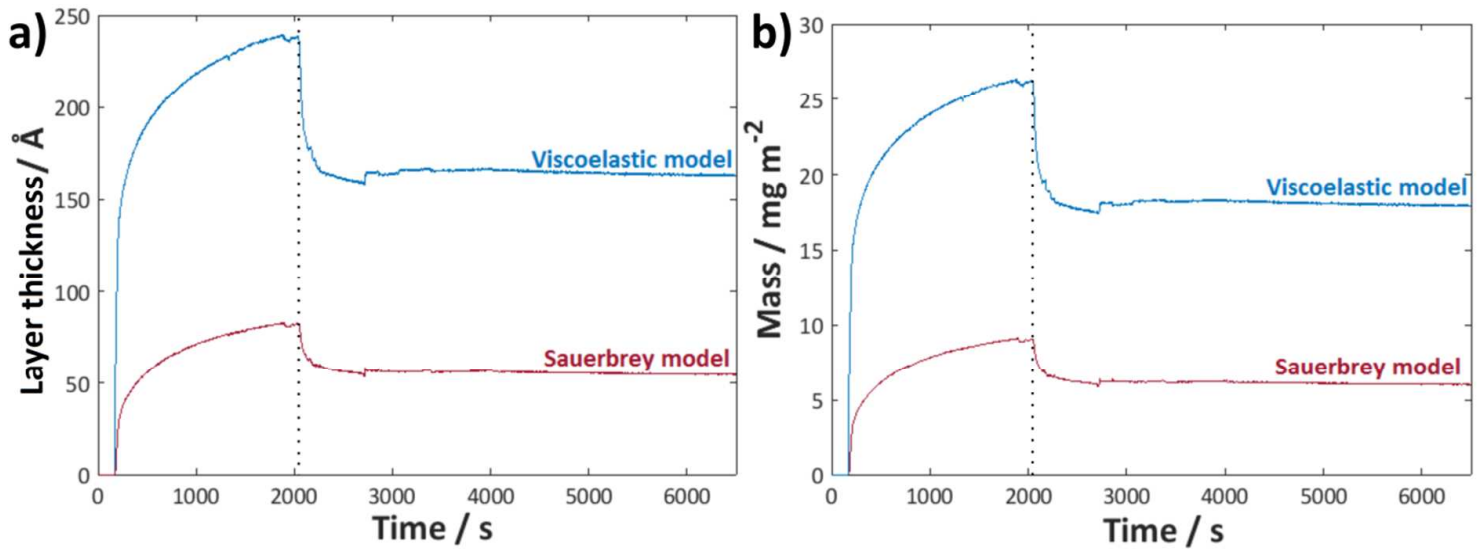

Figure 6. Predicted a) layer thicknesses and b) masses resulting from the Sauerbrey and viscoelastic models, as labelled. The reintroduction of PBS is marked with a dotted line.

Interestingly, the adsorbed mass predicted by both models, as shown in Figure $6 \mathrm{~b}$ is much greater than that calculated from the fit to the neutron data-by well over an order of magnitude in the case of the viscoelastic model. This discrepancy is presumed to arise from 
the acknowledged inability of QCM measurements to differentiate between the protein and associated water in less rigid protein films, which may lead to a significant overestimation of surface loading. The large difference between the two techniques seen here may be partly explained by some level of protein unfolding, as water retention is often greater in such a case $^{48-50}$.

The layer thicknesses shown in Figure $6 \mathrm{~b}$ are calculated using the predicted masses, sensor chip surface area and protein density, and are hence also subject to overestimation. Additionally, as inferred from the NR data, the surface coverage of adsorbed protein is relatively low and so an average thickness is unlikely to correlate well to the actual adsorbed layer thickness, which is best determined using the NR fit. The QCM data are, however, are useful in comparing relative adsorption, as explained below.
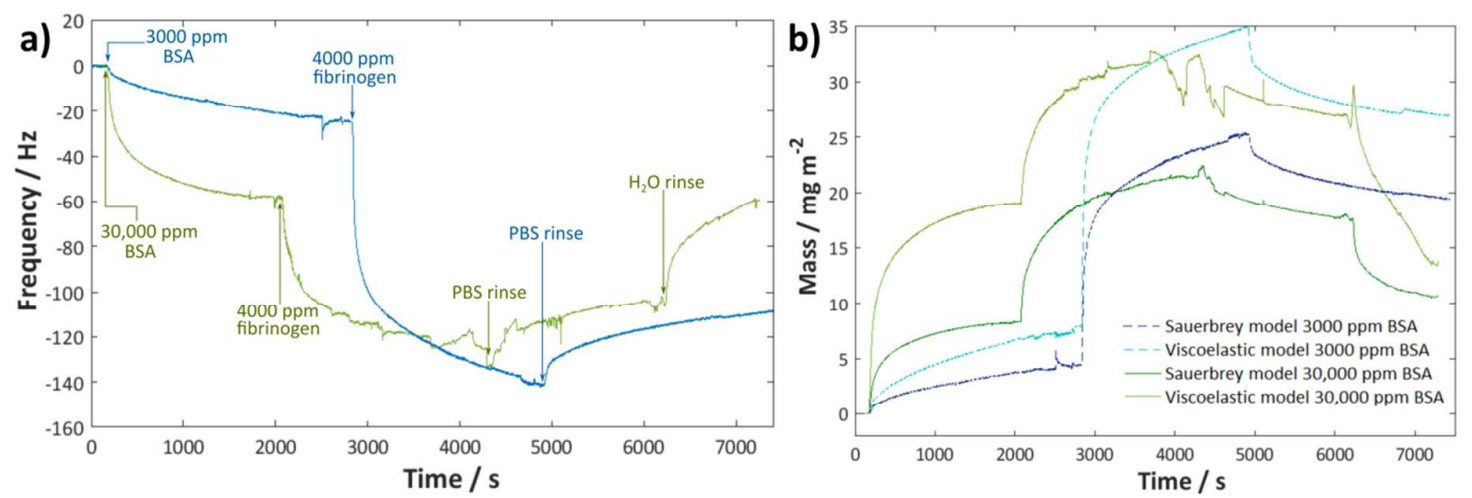

Figure 7a) Comparison of the frequency variation (f3) with time for the $\mathbf{3 1 6}$ stainless steel chips treated with 3000 (green) or 30,000 ppm (blue) BSA prior to 4000 ppm fibrinogen as labelled. b) Modelled adsorbed masses for these data.

To discern the effects of pre-adsorbed BSA on the subsequent adsorption of fibrinogen, two sensors were treated with 3000 and 30,000 ppm BSA respectively and allowed to equilibrate before 4000 ppm fibrinogen/PBS was exposed to the surface. The results are plotted in Figure 7a, with the adsorbed masses predicted using the 
corresponding Sauerbrey and viscoelastic model fits in Figure $7 \mathrm{~b}$. It is clear from Figure 7 that fibrinogen adsorbs to a much lesser extent on the stainless steel surface that has been pretreated with the higher concentration of BSA-almost half, in fact (the precise \% depending on which model type is used to interpret the data). This is in good agreement with the model fits derived from the neutron data above, where a significant reduction in fibrinogen adsorption was seen when the stainless steel surface was pretreated with BSA. In fact, there is an even greater \% reduction seen here than for the neutron data, which may imply that the fibrinogen adsorbing on the pre-treated surface is less prone to structural deformation and hence has less associated water (leading to a lower detected mass).

Around a quarter of the adsorbed protein is removed upon rinsing with PBS for both samples; this is a little less than was removed for the BSA-only sample above (where around a third was removed with a PBS rinse), suggesting the fibrinogen may bond rather more strongly to the stainless steel surface. Previous work showed even less protein was removed when fibrinogen was adsorbed by itself to a stainless steel surface (around $10 \%$ ), which supports this conclusion ${ }^{25}$. The neutron data fits above also estimate around $25 \%$ protein is removed for the BSA-coated sample exposed to fibrinogen (Sample 1), in excellent agreement with this data.

When the rinsing medium was changed to deionized water (for the low BSA concentration only), a greatly increased amount of protein was removed from the surface. This is attributed to the negligible ionic strength of the water and hence increased Debye screening length such that the adsorbed proteins repel each other and are more likely to desorb as a result ${ }^{51}$. 


\section{CONCLUSIONS}

Neutron reflectometry data for a $316 \mathrm{~L}$ stainless steel surface exposed to an approximately physiological concentration of BSA have shown low surface coverage of the adsorbed protein $(<20 \%)$ at an overall thickness of around $80 \AA$, comprising 3 or 4 fitted blocks with slightly different hydration values. Isotherm models fitted to the data suggested monolayer adsorption at lower concentrations with possible bilayer or multilayer at higher concentrations. The high level of water retention observed from the QCM may also indicate some level of protein unfolding on the surface; a viscoelastic model was necessary to fit the QCM data, indicating that the adsorbed protein exists as a diffuse, weakly-bound layer, in agreement with the NR data.

When a solution of $30,000 \mathrm{ppm}$ and $4000 \mathrm{ppm}$ fibrinogen was exposed to the surface pre-treated with BSA, there was an increase in the total amount of protein adsorbed, which then increased again for a solution of just fibrinogen to the value expected when fibrinogen is introduced to a non pre-treated stainless steel surface. This suggests fibrinogen is extremely effective at displacing adsorbed BSA, even at low concentrations.

A second sample was first treated with SDS resulting in around $46 \%$ coverage of the surfactant on the stainless steel surface. As a result, an increased amount of BSA adsorption was observed and an ensuing decrease in fibrinogen adsorption; clearly, the modification of the surface to render it more conducive to BSA adsorption was successful in inhibiting the fibrinogen adsorption. The dramatic decrease (by half) of fibrinogen adsorption seen in the QCM measurements when BSA pre-treatment was increased from 3000 to 30,000 ppm may imply fibrinogen adsorbing on top of BSA is also less prone to unfolding. 
When the surfaces treated with protein solutions were washed in PBS solution, less protein could be removed (around 25\%) if fibrinogen was present than if only BSA had been adsorbed (around a third), further supporting observations that fibrinogen has a greater affinity for stainless steel than BSA once it has gained access to the surface. Hence, although pre-adsorption of BSA is effective in preventing fibrinogen adsorption, over time fibrinogen may still adsorb significantly due to the Vroman effect, unless the BSA is more firmly anchored.

Increasing hydrophobicity of a stainless steel surface by pre-adsorption of a surfactant molecule such as SDS may be an effective method of ensuring improved BSA adsorption and hence inhibition of fibrinogen. However, in all systems studied, a significant amount of fibrinogen was still adsorbed, suggesting these methods are not by themselves fully sufficient in ensuring biocompatibility.

\section{Acknowledgements}

With thanks to Juan Rubio-Lara (Nanoscience, University of Cambridge) for the thin film depositions. This work is based on experiments performed at the neutron reflectometer AMOR at Swiss spallation neutron source SINQ, Paul Scherrer Institute, Villigen, Switzerland.

\section{Supporting Information}

Molar isotherms of fibrinogen and BSA on stainless steel for comparison. QCM raw data with viscoelastic model fits plotted to show goodness of fit.

\section{REFERENCES}


(1) Gorbet, M. B.; Sefton, M. V. Biomaterial-associated thrombosis: Roles of coagulation factors, complement, platelets and leukocytes. Biomaterials 2004, 25, 5681-5703.

(2) Taubert, A.; Mano, J. F.; Rodríguez-Cabello, J. C. Biomaterials Surface Science; WileyVCH Verlag GmbH \& Co. KGaA: Weinheim, 2013.

(3) Sprague, E. A.; Palmaz, J. C. A model system to assess key vascular responses to biomaterials. J. Endovasc. Ther. 2005, 12, 594-604.

(4) Tsai, W.; Grunkemeier, J. M.; Horbett, T. A. Human plasma fibrinogen adsorption and platelet adhesion to polystyrene. J. Biomed. Mater. Res. 1999, 44, 130-139.

(5) Yongli, C.; Xiufang, Z.; Yandao, G.; Nanming, Z.; Tingying, Z.; Xinqi, S. Conformational changes of fibrinogen adsorption onto hydroxyapatite and titanium oxide nanoparticles. J. Coll. Int. Sci. 1999, 214, 38-45.

(6) Ding, Y.-X.; Hlady, V. Competitve adsorption of three human plasma proteins onto sulfhydryl-to-sulfoate gradient surfaces. Croat. Chem. Acta. 2011, 84, 193-202.

(7) Slack, S. M.; Horbett, T. A. Changes in fibrinogen adsorbed to segmented polyurethanes and hydroxyethylmethacrylate-ethylmethacrylate copolymers. J. Biomed. Mater. Res. 1992, 26, 1633-1649.

(8) Horbett, T. A.; Cheng, C. M.; Ratner, B. D.; Hoffman, A. S.; Hanson, S. R. The kinetics of baboon fibrinogen adsorption to polymers in vitro and in vivo studies. J. Biomed. Mater. Res. 1986, 20, 739-772.

(9) Foster, J. R. In Albumin structure, function and uses; Rosenoer, V. M., Oratz, M., Rothschild, M. A., Eds.; Pergamon Press: Great Britain, 1977.

(10) Martins, M. C. L.; Wang, D.; Ji, J.; Feng, L.; Barbosa, M. A. Albumin and fibrinogen adsorption on PU-PHEMA surfaces. Biomaterials 2003, 24, 2067-2076.

(11) Keogh, J. R.; Velander, F. F.; Eaton, J. W. Albumin-binding surfaces for implantable 
devices. J. Biomed. Mater. Res. 1992, 26, 441-456.

(12) Mulvihill, J. N.; Faradji, A.; Oberling, F.; Cazenave, J.-P. Surface passivation by human albumin of plasmapheresis circuits reduces platelet accumulation and thrombus formation. Experimental and clinical studies. J. Biomed. Mater. Res. 1990, 24, 155163.

(13) Kim, S. W.; Lee, R. G.; Oster, H.; Coleman, D.; Andrade, J. D.; Lentz, D. J.; Olsen, D. Platelet adhesion to polymer surfaces. Trans. Amer. Soc. Artif. Int. Organs 1974, 20, 449-455.

(14) Kottke-Marchant, K.; Anderson, J. M.; Umemura, Y.; Marchant, R. E. Effect of albumin coating on the in vitro blood compatibility of Dacron arterial prostheses. Biomaterials $1989,10,147-155$.

(15) Amiji, M.; Park, H.; Park, K. Study on the prevention of surface-induced platelet activation by albumin coating. J. Biomater. Sci. Polym. Edn. 1992, 3, 375-388.

(16) Munro, M. S.; Quattrone, A. J.; Ellsworth, S. R.; Kulkarni, P.; Eberhart, R. C. Alkyl substituted polymers with enhanced albumin affinity. Trans. Am. Soc. Artif. Intern. Organs 1981, 27, 499-503.

(17) Holmberg, M.; Hou, X. Fibrinogen adsorption on blocked surface of albumin. Coll. Surf. B. 2011, 84, 71-75.

(18) Wertz, C. F.; Santore, M. M. Effect of surface hydrophobicity on adsorption and relaxation kinetics of albumin and fibrinogen: single-species and competitive behavior. Langmuir 2001, 17, 3006-3016.

(19) Roach, P.; Farrar, D.; Perry, C. C. Intepretation of protein adsorption: suface-induced conformational changes. J. Am. Chem. Soc. 2005, 127, 8168-8173.

(20) Uyen, H. M. W.; Schakenraad, J. M.; Sjollema, J.; Noordmans, J.; Jongebloed, W. L.; 
Stokroos, I.; Busscher, H. J. Amount and surface structure of albumin adsorbed to solid substrata with different wettabilities in a parallel plate flow cell. J. Biomed. Mater. Res. 1990, 24, 1599-1614.

(21) Hedberg, Y.; Wang, X.; Hedberg, J.; Lundin, M.; Blomberg, E.; Wallinder, I. O. "Surfaceprotein interactions on different stainless steel grades: effects of protein adsorption, surface changes and metal release." J. Mater Sci. Mater. Med. 2013, 24, 1015-1033.

(22) Campana, M.; Hosking, S. L.; Petkov, J. T.; Tucker, I. M.; Webster, J. R. P.; Zarbakhsh, A.; Lu, J. R. Adsorption of bovine serum albumin (BSA) at the oil/water interface: a neutron reflection study. Langmuir 2015, 31, 5614-5622.

(23) Penfold, J.; Thomas, R. K. The application of the specular reflection of neutrons to the study of surfaces and interfaces. J. Phys. Condens. Matter 1990, 2, 1369-1412.

(24) Penfold, J.; Thomas, R. K.; Lu, J. R.; Staples, E.; Tucker, I.; Thompson, L. The study of surfactant adsorption by specular neutron reflection. Phys. B. 1994, 198, 110-115.

(25) Wood, M. H.; Browning, K. L.; Barker, R. D.; Clarke, S. M. Using neutron reflectometry to discern the structure of fibrinogen adsorption at the stainless steel/aqueous interface J. Phys. Chem. B 2016, 120, 5405-5416.

(26) Voinova, M. V; Rodahl, M.; Jonson, M.; Kasemo, B. Viscoelastic acoustic response of layered polymer films at fluid-solid interfaces: continuum mechanics approach. Phys. Scr. 1999, 59, 391.

(27) Björck, M.; Andersson, G. GenX: an extensible X-ray reflectivity refinement program utilising differential evolution. J. Appl. Cryst. 2007, 40, 1174-1178.

(28) Gupta, M.; Gutberlet, T.; Stahn, J.; Keller, P.; Clemens, D. AMOR - the time-of-flight neutron reflectometer at SINQ/PSI. Pramana - J. Phys. 2004, 63, 57-63.

(29) Hughes, A. V. Sourceforge: Downloaded from http://sourceforge.net/projects/rscl/ 
2013.

(30) Sposito, G. The Chemistry of Soils; Oxford University Press: New York, 2008.

(31) Nakagaki, M.; Handa, T.; Shimabayashi, S. S-shaped adsorption isotherms of surface active electrolytes from aqueous solutions. J. Coll. Int. Sci. 1973, 43, 521-529.

(32) Zhu, B.; Gu, T. Surfactant adsorption at solid-liquid interfaces. Adv. Colloid Interface Sci. 1991, 37, 1-32.

(33) Squire, P. G.; Moser, P.; O’Konski, C. T. The hydrodynamic properties of bovine serum albumin monomer and dimer. Biochemistry 1968, 7, 4261-4272.

(34) Gispert, M. P.; Saramago, B.; Serro, A. P.; Colaço, R. Bovine serum albumin adsorption onto $316 \mathrm{~L}$ stainless steel and alumina: a comparative study using depletion, protein radiolabeling, quartz crystal microbalance and atomic force microscopy. Surf. Int. Anal. 2008, 40, 1529-1537.

(35) Pradier, C. M.; Costa, D.; Rubio, C.; Compère, C.; Marcus, P. Role of salts on BSA adsorption on stainless steel in aqueous solutions. 1. FT-IRRAS and XPS characterisation. Surf. Int. Anal. 2002, 34, 50-54.

(36) Singh, M.; Chand, H.; Gupta, K. C. The study of density, apparent molecular volume and viscosity of bovine serum albumin and lysozyme in aqueous and Rbl, Csl and DTAB aqueou solutions at 303.15 K. Chem. Biodivers. 2005, 2, 809-824.

(37) Fukuzaki, S.; Urano, H.; Nagata, K. Adsorption of protein onto stainless-steel surfaces. J. Ferment. Bioeng. 1995, 80, 6-11.

(38) Sakiyama, T.; Tomura, J.; Imamura, K.; Nakanishi, K. Adsorption characteristics of bovine serum albumin and its peptide fragments on a stainless steel surface. Coll. Surf. B 2004, 33, 77-84.

(39) Van Enckevort, H. J.; Dass, D. V; Langdon, A. G. The adsorption of bovine serum 
(41) Hedberg, Y.; Hedberg, J.; Liu, Y.; Wallinder, I. O. Complexation- and ligand-induced metal release from 316L particles: Importance of particle size and crystallographic structure. BioMetals 2011, 24, 1099-1114.

(42) Wertz, C. F.; Santore, M. M. Adsorption and relaxation kinetics of albumin and fibrinogen on hydrophobic surfaces: single-species and competitive behavior. Langmuir 1999, 15, 8884-8894.

(43) Hedberg, Y. S.; Killian, M. S.; Blomberg, E.; Virtanen, S.; Schmuki, P.; Wallinder, I. O. Interaction of bovine serum albumin and lysozyme with stainless steel studied by time-of-flight secondary ion mass spectrometry and X-ray photoelectron spectroscopy. Langmuir 2012, 28, 16306-16317.

(44) Wood, M. H.; Welbourn, R. J. L.; Zarbakhsh, A.; Gutfreund, P.; Clarke, S. M. Polarized neutron reflectometry of nickel corrosion inhibitors. Langmuir 2015, 31, 7062-7072.

(45) Morales-Gil, P.; Walczak, M. S.; Cottis, R. A.; Romero, J. M.; Lindsay, R. Corrosion inhibitor binding in an acidic medium: Interaction of 2-mercaptobenizmidazole with carbon-steel in hydrochloric acid. Corr. Sci. 2014, 85, 109-114.

(46) Molchan, I. S.; Thompson, G. E.; Lindsay, R.; Skeldon, P.; Likodimos, V.; Romanos, G. E.; Falaras, P.; Adamova, G.; Iliev, B.; Schubert, T. J. S. Corrosion behaviour of mild steel in 1-alkyl-3-methylimidazolium tricyanomethanide ionic liquids for $\mathrm{CO}_{2}$ capture applications. RSC Adv. 2014, 4, 5300-5311.

(47) Chan, B. M. C.; Brash, J. L. Adsorption of fibrinogen on glass: Reversibility aspects. J. 
Coll. Int. Sci. 1981, 82, 217-225.

(48) Ouberai, M. M.; Xu, K.; Welland, M. E. Effect of the interplay between protein and surface on the properties of adsorbed protein layers. Biomat. 2014, 35, 6157-6163.

(49) Höök, F.; Kasemo, B.; Nylander, T.; Fant, C.; Sott, K.; Elwing, H. Variations in coupled water, viscoelastic properties and film thickness of a Mefp-1 protein film during adsorption and cross-linking: A quartz crystal microbalance with dissipation monitoring, ellipsometry and surface plasmon resonance study. Anal. Chem. 2001, $73,5796-5804$.

(50) Limson, J.; Odunuga, O. O.; Green, H.; Höök, F.; Blatch, G. L. The use of a quartz crystal microbalance with dissipation for the measurement of protein-protein interactions: A qualitative and quantitative analysis of the interactions between molecular chaperones. S. Afr. J. Sci. 2004, 100, 678-682.

(51) Parkes, M.; Myant, C.; Cann, P. M.; Wong, J. S. S. The effect of buffer solution choice on protein adsorption. Tribol. Int. 2014, 72, 108-117.

TOC GRAPHIC 


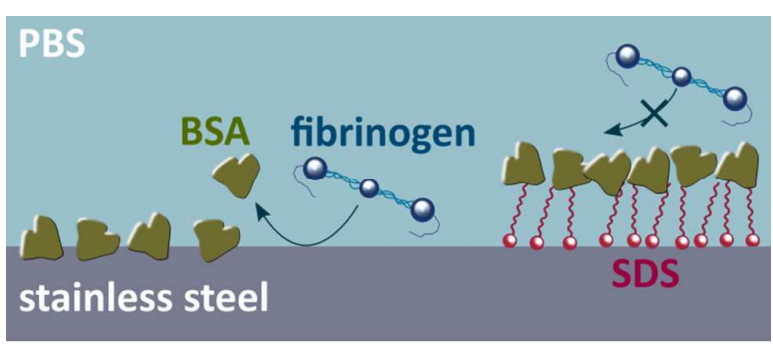

10

11

12

13

14

15

16

17

18

19

20

21

22

23

24

25

26

27

28

29

30

31

32

33

34

35

36

37

38

39

40

41

42

43

44

45

46

47

48

49

50

51

52

53

54

55

56

57

58

59

60 